\title{
Sistemas de Costos de hospitalización en clínicas privadas
}

\section{Raiza Cubillán Muñoz ${ }^{1}$}

\section{Resumen}

Se realiza un estudio comparativo de casos, concretamente dos clínicas privadas, EI Varillal y Centro Médico de Occidente, ubicadas en Maracaibo, Venezuela. Se analizan los sistemas de registro de costos del servicio de hospitalización. El estudio se centra en el análisis de los catálogos de cuentas de ambas clínicas, para establecer la concepción de costos que subyace a la práctica contable y determinar si los registros de costos generan información útil para el control de los mismos y para fijar las tarifas por servicios; los resultados demuestran que los actuales registros presentan serias limitaciones para lograr los fines mencionados, por lo cual se diseña un catálogo de cuentas que intenta superar tales limitaciones.

Palabras clave: Catálogo de cuentas, clínicas privadas, hospitalización, control de costos, tarifas de servicios, atención en salud.

\section{Hospitalization Costs System in Private Clinics}

\begin{abstract}
A comparative case study of two private clinics was made, El Varillal Clinic and The Centro Medico de Occident, both situated in Maracaibo, Venezuela. The mechanisms of hospitalization service cost registration was analyzed. The study focused its analysis on the register of accounts in both clinics, in order to establish the conception of costs which
\end{abstract}

Recibido: $2-3-97$. Aceptado: 18-5-97

1 Contador Público, Docente de la Facultad de Ciencias Económicas y Sociales, Universidad del Zulia. En base al Trabajo preparado para optar al título de Especialista en Contaduría, Mención Costos, del Programa conjunto UCLA-LUZ, con la asesoria de la Prol. Teresa Gamboa C. 
underline the accounting practices; to determine if the registry of costs generates usefull information for the control of the same; and to determine costs per service. The results demonstrate that the present registry process has serious limitations in the achievement of these goals. A registry of accounts was designed to improve this situation.

Key words: Account registry, Private clinics, Hospitalization, Cost control, Service fees, Health service.

\section{Introducción}

A través de esta investigación, se estudiaron los mecanismos de registro de los diferentes elementos de costos correspondientes al servicio de hospitalización prestado en clínicas privadas de medianas dimensiones, cuyo tamaño fluctúa entre 26 y 50 camas.

El servicio de hospitalización es uno de los componentes de la estructura de costos de los servicios de atención médica, prestado tanto por los hospitales como por las clínicas. En los actuales momentos, los costos de atención médica han alcanzado tales niveles que, en muchos casos, resultan inasequibles para amplios sectores de la población, con las imaginables consecuencias para las personas que requieren de dichos servicios. En el caso de las clínicas privadas, el servicio de hospitalización, es uno de los componentes de costos de mayor peso y se considera que cualquier intento que se haga por reducir tales costos sin afectar la calidad del servicio, podria redundar en beneficio para los usuarios.
Por tratarse de un área de investigación relativamente inexplorada y por el corto tiempo estipulado para hacer la investigación, se trabajó con casos seleccionados intencionalmente, a fin de hacer un estudio comparativo de carácter exploratorio, que permita formular hipótesis para estudios futuros más profundos. EI referente empírico abordado fueron las clínicas El Varillal C.A. y Centro Médico de Occidente C.A., usando la información suministrada por su personal directivo, administrativo y contable, así como el análisis de documentos.

Se realizaron entrevistas a miembros de la directiva de las clínicas, al personal de facturación y muy especiaimente a administradores y contadores ${ }^{2}$.

El análisis de documentos constituyó el principal medio de recolección de información, por la naturaleza del objeto de estudio. No pueden comprenderse las características y potencialidades de los registros de costos si no es a partir del catálogo de cuentas y del proceso de contabilización (éste último no fue estudiado en esta oportunidad). Se analizaron tres tipos de documentos: los catálogos de

2 Vale destacar el apoyo y colaboración decidida del Lic. Edgar Zambrano, quien es contador público y ha venido desempeñando el cargo de administrador, primero en la Clínlca El Varilial y actualmente en el Centro Médico de Occidente. 
cuentas, los baremos de servicios y los informes extra-contables de cálculo de costos cama/dia.

Además del análisis de la calidad interna del catálogo, en cuanto a criterios de clasificación y pertinencia de la denominación de los grupos, subgrupos y cuentas, se realizaron dos comparaciones básicas: en primer lugar se contrastó la sección de egresos de los catálogos con los respectivos baremos, a fin de establecer la potencialidad de los registros parafijar los precios de los servicios; y en segundo lugar se compararon los catálogos con los renglones utilizados en las estimaciones extra-contables realizadas para calcular el costo cama/dia.

Adicionalmente, $y$ de manera permanente, se realizó un análisis teóricoconceptual para establecer la validez práctica de los conceptos generales de costos y afines, relacionados con el objeto de estudio.

\section{Costos en función de competitividad en clínicas privadas}

La crisis económica que vive la sociedad venezolana ha afectado la prestación de los servicios de salud, tanto en el sector público como en el privado. Se han deteriorado seriamente las instituciones de salud pública, con lo cual resultan más necesarios los servicios privados de salud, tanto para los sectores sociales que poseen capacidad de compra de los mismos, como para grandes instituciones y organizaciones gremiales que contratan tales servicios para su personal o afiliados.
Debido a los avances tecnológicos, los servicios médicos se han vuelto cada vez más complejos y en consecuencia, sus costos se han incrementado de tal manera que llegan a ser inasequibles a una buena parte de la población. Esto es más grave en las clínicas privadas, debido al objetivo económico perseguido por las mismas; su sobrevivencia está directamente relacionada con el logro de beneficios y por lo tanto, con la correlación favorable ingresos/costos.

De acuerdo con declaraciones de los directores de clínicas privadas de $\mathrm{Ma}$ racaibo, debido a la crisis y a las medidas económicas, han visto reducir la demanda de servicios médico-asistenciales privados en un $75 \%$; por lo tanto, deben idear fórmulas, cada vez más creativas, para mantenerse competitivas en el mercado. Por otro lado, ante las denuncias por cobros considerados excesivos, la Asamblea Legislativa del Estado Zulia, se planteó investigar los costos de la medicina privada. (Panorama, 30 y 31/05/96). Aunque el $75 \%$ luzca un tanto exagerado, es indudable que los sectores medios (profesionales y medianos empresarios) han visto disminuir su status socioeconómico y por lo tanto, inevitablemente, han debido reducir o modificar sus hábitos de consumo de bienes y servicios.

En estas condiciones, las clínicas privadas están conscientes de que necesitan desarrollar estrategias de reducción de costos que les permitan ofertar sus servicios a precios competitivos y accesibles para un número importante de potenciales usuarios.

Por otra parte, los procesos de reforma de la administración pública en 
América Latina, sugieren la tendencia a la privatización de los servicios de salud, como puede observarse en las experiencias de paises como Chile y Colombia, asi como en las propuestas que actualmente se discuten en varios países. En Colombia, por ejemplo, se ha realizado una transformación total del sistema de salud, que ha obligado a los hospitales y clínicas privadas a competir entre si, por la contratación de servicios con empresas aseguradoras; éstas venden servicios de medicina prepagada de acuerdo a un plan oficial obligatorio de salud, con la posibilidad de ofertar planes complementarios, relativos a mejores condiciones de hotelería hospitalaria o al uso de servicios diagnósticos y clínicos de alta tecnología. (Gamboa y Rangel, 1996)

La Ley Orgánica Marco de Seguridad Social, aprobada por el Congreso de la República, generará la privatización de los servicios de salud, separando las funciones de aseguramiento y prestación del servicio; dicha Ley prohibe que los aseguradores (empresas de seguros y de medicina prepagada) sean propietarios de las instituciones prestadoras de servicios. Esto significa que los servicios serán contratados por las empresas aseguradoras, con un probable dominio respecto a la fijación de tarifas, como lo sugiere la experiencia de Colombia.

Es indudable que en las condiciones actuales o en el futuro, de acuerdo a las reformas posibles, la racionalización de costos se imponga como una necesidad insoslayable. En Colombia, la presión por la reducción de costos de los servicios médicos en general, constituye la preocupación central en las instituciones públicas y privadas de salud. (Jaramillo, 1994)

Aunque el problema de costos no es sustancialmente un problema contable, sino operativo, sin lugar a dudas, la existencia de registros adecuados que generen informes pertinentes y oportunos constituye un apoyo vital, aunado a la capacidad del nivel gerencial para utilizarlos como base para la toma de decisiones. La contabilidad de costos es una herramienta de apoyo en la toma de decisiones, pues los costos juegan un papel muy importante, tanto en la planificación y control, como en la determinación de utilidades. En tal sentido, Robert Kaplan (cit. por González et al, 1996) afirma que las empresas de servicios deben analizar, incluso más seriamente que las empresas de manufactura, el costo real de sus servicios.

\section{Concepción de costos subyacente en las clínicas estudiadas}

En un primer intento de conocer los procedimientos usados para el registro de los costos incurridos por las clínicas EI Varillal -CEV- y Centro Médico de Occidente -CMO-, a causa de la prestación de servicios de salud, se llegó a la conclusión de que en ninguna de ellas existe un sistema riguroso de acumulación de costos.

Ante esta situación, se plantea la posibilidad de utilizar el catálogo de cuentas como base para: a) Determinar la concepción de costos que subyace a la práctica contable, y b) Analizar la calidad potencial de los registros contables en fun- 
ción de su posibilidad para suministrar información que facilite la determinación de los precios de los servicios prestados y el control de los costos respectivos.

Cabe destacar que el análisis se limitó al grupo de los egresos, y dentro de éste, se hizo énfasis en los subgrupos relacionados con la prestación del servicio de hospitalización en habitación normal.

En razón de que la exploración preliminar evidenció que ninguna de las dos clínicas cuenta con un sistema riguroso de costos, se consideró necesario dilucidar cual es la concepción de costos que prevalece en la práctica contable y gerencial.

\section{Concepción funcional de costos}

Se aborda, en primer lugar, el análisis del catálogo de cuentas de la CEV, clínica cuyas caracteristicas son las siguientes: Fue creada en 1988 como una asociación cooperativa sin fines de lucro, y transformada en compañía anónima en 1990; el $1^{\circ}$ de septiembre de 1997 surge otro cambio en su dirección, aparece la figura de la Operadora El Varillal, la cual se encarga de controlar las operaciones y los egresos de la clínica, conservando ésta la actividad de atención médica. La capacidad funcional de la clínica es de 26 camas; presta servicios de hospitalización, cuidados intensivos, quirófano, emergencia, rayos $x$, laboratorio, anatomía patológica, ultrasonido, terapia respiratoria, sala de partos, sala de quemados, retén, banco de sangre (contratado), farmacia; cuenta con una dirección de administración integrada por los departamentos de recursos humanos, contabilidad, facturación y cobranzas, caja, vigi- lancia, depósito, servicios generales y compras y con las secciones de saneamiento y mantenimiento.

El análisis evidencia que la concepción de costos que subyace en la CEV es el enfoque tradicional funcional, en el cual los costos o egresos se agrupan de acuerdo a las grandes funciones organizacionales, sin ninguna vinculación con los ingresos que deben generar, ni con los servicios que los causan.

Según Irarrázabal $(1991,26)$ la estructura organizacional funcional de una empresa manufacturera puede comprender las áreas comercial, administrativa, $\mathrm{fi}$ nanciera y el área de operaciones; esta última incluye producción, mantenimiento e ingenieria. Si adaptamos este planteamiento a la estructura de una clínica, bastaría considerar que la función de producción corresponde a la prestación de servicios de salud, para lo cual se viene manejando recientemente el término "servucción".

Sin embargo, como plantean Irarrázabal $(1991,26)$ y Polimeni $(1994$, 715), toda empresa se puede organizar de acuerdo a sus necesidades y a las características propias de su giro, existiendo otros tipos de estructuras organizacionales además de la funcional: por productos (o servicios), por clientes, por mercados, por canales de distribución, por territorios, por proceso, etc.

De acuerdo con su concepción funcional, en el Catálogo de Cuentas de la CEV se observan dos grandes grupos de egresos: Gastos Operativos y Gastos Generales y de Administración. Además, tenemos el grupo de Egresos Financieros, dentro del bloque Ingresos y Egresos Financieros (6000), diferenciado total- 
mente de los bloques ingresos (4000) y Egresos (5000).

El primer grupo de egresos, denominado Gastos Operativos, se corresponde con los desembolsos relacionados con la prestación de los distintos servicios de la CEV. La denominación de los Gastos Operativos no corresponde a ninguna función específica de la organización hospitalaria; hay que tener en cuenta que las clínicas son empresas de servicios, y que su estructura operativa y de costos es diferente a la de las fábricas o empresas industriales.

Si el objeto de estudio fuera una empresa industrial, la función producción estaría bien definida; es a través de ella que se generan los desembolsos relacionados, directa o indirectamente, con la fabricación de los productos, denominados costos de producción. De modo que, en el caso de la CEV, los Gastos Operativos mencionados, que corresponden a la prestación de servicios de salud, se expresan en forma similar a las erogaciones derivadas de la función producción, en la empresa industrial.

En cuanto al grupo Egresos Generales y de Administración, aún cuando es bastante amplio, en su mayoría las cuentas se corresponden con la tradicional función de administración. Por último, tenemos el subgrupo Egresos Financieros, el cual recoge los intereses bancarios y comerciales, pudiendo corresponderse con la función financiera, la cual ha adquirido importancia en los últimos años, al reducirse el crédito estatal barato.

En consecuencia, la CEV se limita a aplicar un enfoque tradicional funcional. Según Ramírez Padilla (1994, 285-6), un sistema contable que sólo genere infor- mación general sobre los resultados obtenidos por funciones en la empresa, no permite llegar al verdadero problema, no pudiéndose responsabilizar a las personas por los errores cometidos, ni por los éxitos obtenidos. De allí se origina la necesidad de un sistema contable que informe la actuación de las diferentes áreas o unidades de la organización, al frente de las cuales está un responsable de los gastos o ingresos que alli se generan, o de ambos simultáneamente, de manera que los superiores estén informados sobre los resultados de la gestión y puedan aplicar las medidas que consideren convenientes.

\section{Concepción de costos por áreas de responsabilidad}

A diferencia del caso que se acaba de analizar, el CMO maneja una concepción de costos vinculada a la contabilidad por áreas de responsabilidad. EI CMO es una clínica de mayor complejidad y con mucho más tiempo en el desempeño de sus funciones. Su capacidad funcional es de 50 camas; presta servicios de hospitalización, cuidados intensivos, quirófano, banco de sangre, emergencia, rayos $x$, laboratorio, anatomia patológica, farmacia, fisioterapia, uttrasonido, sala de partos, sala de yeso, retén, sala de diálisis; la dirección de administración cuenta con los departamentos de facturación, cobranzas, admisión, contabilidad e informática.

En el catálogo de cuentas del CMO se pudo observar una sola sección de egresos, denominada Costos de los Servicios Prestados, la cual está conformada por 13 subgrupos a saber: Costo de Hos- 
pitalización, Unidad de Cuidado Intensivo, Quirófano, Banco de Sangre, Emergencia, Rayos $X$, Laboratorio, Anatomía Patológica, Farmacia, Suministro, Fisioterapia, Ultrasonido y Administración.

Del estudio de los subgrupos mencionados, se deduce que la concepción de costos subyacente va más allá del enfoque tradicional por funciones. Se observa que el registro de egresos del CMO fue dividido en varias áreas de acuerdo a los distintos servicios prestados, ratificado esto por el hecho de que el único grupo de egresos que contiene el catálogo de cuentas se denomina Costo de Servicios Prestados. Sin embargo, es de notar que algunos servicios no cuentan con su correspondiente control en el catálogo de cuentas, tal es el caso de sala de partos, sala de yeso, retén y sala de diálisis .

Se observa claramente que en el catálogo de cuentas del CMO, subyace el enfoque de costos presente en la contabilidad por áreas de responsabilidad, ya que los egresos fueron divididos de acuerdo a las distintas áreas de servicios que los causan.

Cada una de estas áreas de servicios, pudiera considerarse un centro de costos, que según Polimeni $(1994,721)$ es un centro de responsabilidad, al cual se le asigna (delega) control sólo sobre los costos en que incurre, aunque no tenga control sobre las ventas o actividades de mercadeo. Los mismos autores, definen los centros de responsabilidad como segmentos (partes de una organización o líneas de actividad) en los cuales se divide una empresa, con gerentes que tienen responsabilidad sobre áreas (actividades) especificas. Existen otros dos tipos de centros de responsabilidad: el centro de utilidades (que ejerce control tanto sobre los costos causados como respecto a la generación de ingresos) y el centro de inversión (que controla no sólo la generación de ingresos y costos, sino también la adquisición de activos del centro de inversión).

Es evidente que los criterios de registros de costos por áreas de actividad aportan información discriminada más pertinente para ejercer control sobre los egresos, correlacionarlos con los ingresos y facilitar la racionalización de costos; esto es imposible si todos los costos están registrados en un número de cuentas funcionales, como en el primer caso analizado.

\section{Utilidad potencial de los registros de costos}

Según Irarrázabal $(1990,19)$ y Ramírez Padilla (1994, 285-286), la contabilidad, en su forma clásica, está orientada a generar información sobre los resultados obtenidos por funciones en la empresa. Un ejemplo, lo tenemos en el estado de resultados, el cual sirve como marco de referencia para comparar con los gastos presupuestados para las funciones de ventas y administración. En este sentido, los sistemas contables financieros fallan como sistemas de información gerencial o de toma de decisiones, porque: a) No proveen información para un control operacional, por tanto los gerentes de producción y de servicios no saben si están reduciendo costos y aumentando su productividad; b) No suministran información para una determinación realista de los costos de los productos fabricados o de los servicios prestados; c) No permiten 
analizar el comportamiento y el desempeño de las distintas áreas.

En opinión de Horngren et al (1996, 4), el sistema de contabilidad debe proporcionar: a) Reportes internos para planeación y control de costos de operaciones y evaluación del desempeño del personal y sus actividades; b) Informes internos sobre la rentabilidad de productos, servicios, clientes, canales de distribución, etc., los cuales son útiles en la toma de decisiones respecto a la asignación de recursos y en algunos casos para decidir precios; c) Informes internos para decisiones estratégicas y tácticas en cuanto a formulación de politicas globales y planes a largo plazo, desarrollo de nuevos productos y servicios, investigación de equipos, y actuaciones especiales; d) Reportes externos mediante los estados financieros dirigidos a los inversionistas, gobierno, entidades financieras, etc.

Una manera novedosa de preparar y utilizar la información es presentado por Khadem y Lorber $(1994,33)$. Según los autores, los gerentes deben recibir sólo la información esencial, es decir, la que necesitan para: a) concentrarse en la información clave acerca de sus actividades; b) resaltar las buenas y malas noticias sobre lo que hacen; y c) recibir las buenas y malas noticias acerca de lo que hace el personal a su cargo. Esta información se les debe presentar a los gerentes en tres informes de una página: el Informe de Enfoque, el de Retroalimentación y el de Gerencia; dichos informes deben permitir realizar comparaciones entre el status y las metas (minimas, satisfactorias y sobresalientes), asi como conocer los facto- res de éxito y los problemas más importantes.

\section{Limitaciones prácticas del enfoque funcional}

El desconocimiento o subestimación de la importancia de la Contabilidad de Costos, se evidencia inicialmente en el hecho de que en la CEV, el catálogo no utiliza expresamente el término costos; todas las erogaciones son consideradas egresos o gastos. En este sentido, se estima necesario hacer algunas considera. ciones tendientes a establecer la distinción entre costos y gastos.

En algunos casos los contadores distinguen entre los costos del producto y los costos del período. Los costos del producto 0 costos inventariables, son erogaciones asociadas con la adquisición y conversión de materiales y otros insumos de producción en productos para su venta; son asignados a inventarios cuando se incurre en ellos y se convierten en gastos (como costo de las mercancías vendidas) sólo cuando los productos se venden. Conforme a los principios contables generalmente aceptados, los costos inventariables están restringidos a los costos de producción para una compañia del sector industrial. En cambio, los costos del período siempre se llevan a gastos en el mismo período en el que se incurren, por no tener relación directa con las actividades de producción; por lo tanto no se adicionan al valor de los productos fabricados, es decir, no pasan por una etapa de inventario (Horngren et al, 1991, 34; 1996, 37)

Según Ortega $(1990,20)$, a los costos del producto se les conoce como cos- 
tos de producción o Costos propiamente dichos, usando el término en un sentido más limitado; los costos del período son designados costos de distribución, administración y financiamiento $y$, en un aspecto más limitado también, se los denomina Gastos.

Según Horngren et al $(1996,35)$, las empresas de servicios - tal es el caso de las clínicas - proporcionan a sus clientes servicios o productos intangibles, los cuales no pueden almacenarse, $y$, por tanto no tienen inventarios ni costos inventariables. Los autores se refieren a inventarios de productos tangibles disponibles para la venta, sin embargo, es conveniente mencionar que en el caso de la clínicas existen inventarios necesarios para la prestación de servicios: Medicinas, materiales médico hospitalarios, suministros no médicos, materiales de limpieza, lencería, partes y repuestos para reparación y mantenimiento, suministros de oficina, etc.

Si bien es cierto que en las clínicas no puede hablarse propiamente de costos inventariables, puede igualmente distinguirse entre las erogaciones generadas directa o indirectamente durante el proceso de prestación de los servicios (costos), y las otras erogaciones causadas por aquellas secciones que colaboran para que las áreas prestadoras de servicios puedan desarrollar sus actividades (gastos). Al respecto existe una nueva concepción de costos - la cadena de valores -, sobre la cual se hablará más adelante.

Aún cuando se desee clasificar las erogaciones de acuerdo a su posible identificación con el proceso de prestación de los servicios, es necesario tener en cuenta cual es la finalidad que se persigue al determinar el costo de los servicios. Aquí es oportuno mencionar lo expresado por Horngren et al (1996, 45): EI costo de un producto es la suma de los costos asignados al mismo con un propósito especifico; identifica tres propósitos diferentes:

- Determinar el precio de productos con énfasis en algún producto o servicio clave. Se incluyen los costos de todas las áreas de la cadena de valores necesarias hasta llevar el producto al cliente.

- Facilitar la contratación con oficinas gubernamentales. Estas dependencias suelen establecer directrices detalladas sobre los importes permisibles y no permisibles en las partidas del costo de productos.

- Producir reportes financieros externos. De acuerdo a los principios de contabilidad generalmente aceptados, para reportes externos, sólo se asignan a los productos los costos de producción.

Para Shank y Govindarajan (1995, 16), la cadena de valores, en una empresa de cualquier área, es el conjunto interrelacionado de actividades creadoras de valor, que se extienden durante todos los procesos, y que van desde la consecución de fuentes de materias primas para proveedores de componentes hasta que el producto terminado se entrega finalmente en las manos del consumidor. Según Porter (cit. por Shank y Govindarajan, 1995, 70), estas actividades son: Obtención de materias primas, investigación y desarrollo, producción, mercadeo, distribución y servicio al cliente. 
Estas actividades no están limitadas a las empresas industriales. Según Horngren et al (1996, 2-3) la cadena de valores es la secuencia de funciones comerciales, y su utilidad (funcionalidad) se añade a los productos o servicios de una organización; al definir cada una de las funciones (actividades) de la cadena de valores, se refiere a productos, servicios o procesos.

En opinión del mismo autor, ${ }_{1}(1996$, 124), es importante que las organizaciones tengan la capacidad de combinar costos de todas las áreas de la cadena de valores; por ejemplo, en relación a la estrategia de precios de una firma de auditores (empresa de servicios), al hacer las estimaciones de costos es esencial que los costos de investigación y desarrollo de tecnologías actualizadas de auditoría, y los costos de mercadeo estén cubiertos por los ingresos totales recibidos por la empresa. Un sistema de costeo que combine los costos de todas las áreas funcionales de la cadena de valores de la empresa, puede ser de gran ayuda para las decisiones de los administradores sobre precios y sobre cuales productos, servicios o clientes deben enfatizar.

Es indudable, que las consideraciones realizadas en torno al costeo de productos o servicios para distintos propósitos, y especialmente lo relativo a la cadena de valores, son aplicables a las clínicas, como empresas de servicios.

En cuanto a la utilidad del catálogo de cuentas de la CEV, observamos que sólo consta de 3 grupos de egresos: Gastos Operativos, Gastos Generales y de Administración y Egresos Financieros. Estudiando con cierto detenimiento el grupo Gastos Operativos, encontramos algunos aspectos que vale la pena mencionar.

La clínica registra los desembolsos generados por todos los servicios que presta a sus pacientes, pero no hace una separación de los mismos. Esto no permite que se puedan determinar los costos de algún servicio en particular, por ejemplo, hospitalización, emergencia, quirófano, unidad de cuidados intensivos (UCl), etc.

No existe correspondencia entre las cuentas del catálogo y los distintos conceptos objeto de facturación listados en el Reporte de Servicios que maneja la sección de facturación y cobranzas; ésta, a los efectos de facturar, dispone de un baremo independiente de los costos reales. En este sentido, los costos calculados a través del catálogo de cuentas no son de utilidad si se quisiera determinar si las tarifas cobradas son rentables; tampoco serán útiles, si por razones de competencia es necesario mantener algunas tarifas bajas, en cuyo caso se deben tomar acciones para reducir los costos.

En algunos casos no es pertinente la ubicación de ciertas cuentas en un subgrupo. Un ejemplo es el subgrupo Materiales, el cual recoge cuentas de naturaleza tan diferente como: Medicinas, utensilios de cocina, lencería, al lado de otras como, material de limpieza, materiales y repuestos de mantenimiento.

Un caso similar ocurre con el subgrupo Otros Gastos, el cual contiene varias cuentas referentes a alquiler, que pudieran conformar un subgrupo específico. Nutrición y Dietética tampoco debería aparecer dentro de Otros Gastos, por su significación dentro de los costos de hospitalización; ocurre algo similar con otras 
cuentas del mismo subgrupo, lo cual no se justifica, considerando que la estructura del catálogo (99 posibles subgrupos) permite hacer una clasificación más precisa de las cuentas. La denominación de los subgrupos debe ser más específica; de esta manera se evita que se reúnan rubros de distinta naturaleza. Esto debe tomarse en consideración, al momento de clasificar las cuentas.

En cuanto al subgrupo Descuentos y Devoluciones, con su única cuenta Descuentos en Compras, no es pertinente su inclusión en el grupo de los egresos; su saldo siempre acreedor, distorsiona el valor de los egresos. Si se considera necesario llevar el control de los descuentos en compras, como éstas registran en la CEV en el subgrupo Inventarios, se podría crear una cuenta complementaria en el mismo subgrupo, cuyo saldo es acreedor, ajustando de paso el valor de los mismos.

El subgrupo Reparación y Mantenimiento debería contemplar la cuenta Materiales y Repuestos de Mantenimiento, agrupada en el subgrupo Materiales; también una cuenta para sueldos del personal que realice las labores de reparación y mantenimiento, la cual no aparece explicitamente en algún subgrupo.

Hay otro aspecto, relativo al catálogo de cuentas de la CEV, que merece ser mencionado: su estructura conlleva registros contables con poca utilidad práctica al momento de calcular el costo y beneficio de cada servicio 0 , específicamente, al determinar el costo cama-paciente de hospitalización, el cual es definido como el costo incurrido en cada período de 24 horas durante el cual la cama esté ocupada por un paciente; este térmi- no es equivalente a costo cama/día ocupada. Un trabajo para determinar estos costos fue realizado por Milagros Rondón, pasante de LUZ, en 1995; es evidente que los datos usados no fueron tomados de los registros contables, por cuanto recurrió, entre otras cosas a: Información suministrada por el departamento de personal, para determinar los sueldos y salarios de enfermería; elaboración de cuadros, basándose en la Ley Orgánica del Trabajo, para calcular los beneficios sociales establecidos en la misma; información facilitada por el departamento de servicios generales para estimar el costo de lencería. De modo que algo tan importante para una clínica como el costo cama/día de hospitalización, no se pudo calcular a partir de los registros" contables existentes.

\section{Factores que restringen la utilidad del enfoque por áreas de responsabilidad}

En este aparte se retomará el análisis del catálogo de cuentas del $\mathrm{CMO}$, limitado al rubro de los egresos, el cual está conformado por 13 grupos, que se corresponden con otras tantas áreas de la clínica: Costos de Hospitalización, Unidad de Cuidado Intensivo, Quirófano, Banco de Sangre, Emergencia, Rayos $X$, Laboratorio, Anatomía Patológica, Farmacia, Suministros, Fisioterapia, Ultrasonido y Administración.

Revisando el actual catálogo de cuentas, encontramos que la estructura de los códigos, está conformada por un máximo de 10 dígitos. De la revisión del mismo se deduce que hay algunos dígitos innecesarios, que lo hacen menos fá- 
cil de manejar. Por ejemplo, el dígito usado para identificar un solo grupo, Costo de Servicios Prestados, no es necesario, ya que todos los egresos aparecen dentro de este gran grupo; adicionalmente, parece excesivo reservar 4 dígitos (9999 posibilidades) para el último nivel, el de las subcuentas.

Las siguientes son algunas observaciones surgidas del análisis del catálogo de cuentas del CMO, el cual se realizó fundamentalmente en torno al grupo Costos de Hospitalización, cuya denominación luce no pertinente, ya que cada uno de los otros grupos pareciera identificar un área de la Clínica, y sólo en el caso de Hospitalización se le antepone la expresión "costo de". Además, el primer subgrupo de Costo de Hospitalización se denomina Hospitalización, inconveniente porque casi todas las cuentas de esta división son sueldos, salarios, honorarios y otras remuneraciones del personal.

Algunas cuentas no tienen una adecuada ubicación dentro del respectivo subgrupo. Como ejemplo podemos mencionar el subgrupo Hospitalización, el cual incluye casi exclusivamente costos relativos al personal, sin embargo, contiene también la cuenta Alimentación Pacientes.

En los demás grupos correspondientes a los costos de áreas funcionales, el primer subgrupo es denominado Costo de Operaciones, lo cual también se considera no pertinente ya que los costos de operaciones no se limitan a los egresos relacionados con el personal, que son los que representan las cuentas que contiene.

En el subgrupo Materiales y Suministros, aparece la cuenta Exámenes Ex- ternos, usada para registrar los desemboisos efectuados cuando se requiere de exámenes en laboratorios ajenos a la Clínica; tales desembolsos no son propiamente por materiales y suministros. También se observa dentro de Materiales y Suministros, la cuenta Lavandería; sin embargo, este es un servicio contratado cuyo costo debería aparecer, en todo caso, en el subgrupo Contratos y Servicios.

En ninguno de los subgrupos del área de Hospitalización, se observan cuentas para registrar lo siguiente: Depreciación de los equipos, mobiliario, planta física, etc., que son utilizados para prestar este servicio; desembolsos por concepto de saneamiento; el consumo de esta área por concepto de servicios públicos. Las cuentas que recogen las erogaciones de este tipo, incurridas en las distintas áreas de servicios, se encuentran reunidas en el grupo correspondiente al área de Administración, aunque el consumo mayor se realiza en la prestación de los servicios. En este caso sería conveniente desarrollar criterios de aplicación de estos egresos. Según Temes $(1994,47)$ los registros contables deben estar dirigidos a la evaluación, de manera directa, o mediante criterios de reparto preestablecidos, de los hechos económicos internos de los hospitales y clínicas (consumo de materiales, gastos de personal) para conocer el gasto y beneficio de cada uno de sus servicios.

Tampoco se observa, en el grupo Costos de Hospitalización, cuentas especíticas para registrar algunos desembolsos que genera un paciente internado por concepto de: Admisión, facturación y cobranzas, uso de televisor y nevera, etc. 
Serla conveniente que los costos de alimentación, por su importancia, se registren en un subgrupo específico, donde además de los honorarios de la nutricionista, se carguen los desembolsos por concepto de alimentos (si los hubiera, ya que actualmente es un servicio contratado que usa el área de la cocina y sus equipos), asi como otros insumos, y depreciación de equipos y de planta física.

Si comparamos las partidas del catálogo de cuentas con los conceptos facturables que aparecen en el Baremo de Servicios del área de Hospitalización, se observa que no se corresponden; al igual que en la CEV, no son los registros contables la fuente de información para confirmar si las tarifas del Baremo son rentables o no; o para establecer algún correctivo para la reducción de costos, en aquellos casos que por razones de competencia, sea necesario reducir algunas tarifas.

Otro aspecto que merece mención, está referido a lo siguiente: Aún cuando las distintas erogaciones causadas por los servicios prestados, están separadas por centros de costos, se requiere de una reagrupación de los desembolsos, al mismo tiempo que mayor especificación para efectos de análisis, control y facturación. Esto lo evidencia un estudio del costo cama/dia de hospitalización, realizado por el Lic. Edgar Zambrano, Coadministrador del CMO, para lo cual tuvo que recurrir a información extra contable: La nómina sivió de base para calcular los costos del personal directo e indirecto y del médico residente en piso; los contratos de servicios fueron usados para determinar el costo de los mismos; partiendo de los consumos reales de toda la clinica se estimaron los costos de los servi- cios públicos correspondientes a hospitalización; con la información relativa a los activos asignados al área de hospitalización se elaboraron cuadros para calcular los costos de depreciación. Igual que en la CEV, la información contable no es útil para efectuar estos cálculos, a pesar de que tiene un mayor grado de discriminación.

\section{Catálogo de cuentas de egresos propuesto}

La contabilidad moderna requiere de un gran número de cuentas, para solucionar las extensas tareas de control estadístico que se le imponen; es preciso establecer tantas cuentas como sea necesario, las cuales deberían tener un contenido y una interdependencia correlativa que sivan al objetivo que se persigue. Los actuales sistemas computarizados de contabilidad facilitan el manejo de códigos complejos. El sistema de cuentas resultante, encuentra la construcción más racional posible a través del catálogo de cuentas.

Un catálogo relaciona las cuentas referentes a los activos, pasivos, capital contable, ingresos y egresos de una organización, con números equivalentes o códigos, los cuales facilitan los registros contables. La clasificación de las distintas erogaciones, debe cumplir las siguientes condiciones, las cuales son propias de todo proceso de clasificación: a) Exclusividad, es decir, no deben surgir dudas acerca de la inclusión de una erogación en el subgrupo correspondiente. Esto exige que cada grupo debe estar suficientemente separado de los demás y debe tener una denominación que lo identifi- 
que plenamente; b) Exhaustividad, 10 cual significa que se deben prever todas las posibles erogaciones que razonablemente puedan presentarse; c) Flexibilidad, requisito que permite agregar nuevos rubros no previstos, sin que eso implique reestructurar todo el catálogo.

En base al análisis realizado se formaliza la propuesta de un catálogo de cuentas que intenta superar las deficiencias identificadas. Se diseñó sólo una sección del catálogo de cuentas, la referida a los egresos de manera general, y de manera particular al área de hospitalización en habitación normal. Este esquema sería el punto de partida, que serviría posteriormente, una vez estudiadas las demás áreas de servicios, para la estructuración de un catálogo que contenga no sólo los egresos, sino también los ingresos.

Previo a la estructuración de un catálogo de cuentas, para un hospital o clínica, es necesario hacer un análisis de todas las divisiones o áreas de actividad establecidas en dichas instituciones, o factibles de ser organizadas, con el fin de determinar costos, las cuales reúnen desembolsos de diferente naturaleza realizados para conseguir un objetivo común. Estas divisiones suelen denominarse: Sectores, secciones, centros de costos, 0 áreas de servicios.

Si se relaciona la función que desempeña una sección con el producto fabricado o servicio prestado, se observa que algunas secciones intervienen directamente en el proceso de fabricación o de prestación de servicios. Otras en cambio, no agregan nada directamente al producto o servicio; su función es la de prestar servicio a las primeras para que alcancen el objetivo propuesto. Las primeras son las que se seleccionan como centros de costos, por cuanto tienen sus ingresos y costos propios. Las segundas son secciones auxiliares o de servicios administrativos y sus costos se imputan en forma proporcional a las definidas como centros de costos.

En los hospitales y clínicas, los centros de costos serian las secciones que prestan directamente un servicio asistencial de salud y por lo tanto, generan un ingreso: servicios de hospitalización y servicios complementarios de diagnóstico y tratamiento.

Las otras secciones que no prestan un servicio asistencial de salud, sin embargo, son indispensables para el buen funcionamiento de los servicios asistenciales; sus costos deben ser cargados a estos últimos en proporción al beneficio recibido. Tal es el caso de historias médicas, facturación, mantenimiento, saneamiento, lavandería, lencería, etc.

Según Temes et al $(1994,51)$, es fundamental que exista una conexión entre la organización estructural del hospital o clínica y la información contable; para ello debe tenerse en cuenta un organigrama funcional con representación real de las dependencias organizativas de cada división y, en consecuencia, cada sección o área de servicios ha de tener su responsable.

El catálogo de cuentas debe facilitar el registro separado de los costos controlables, por los cuales hay que rendir cuentas. Esto permite determinar qué sección está generando mayor beneficio o pérdidas; se establecen así responsables y se minimizan errores, desperdicios, etc., ya que el responsable de la 
sección estará comprometido a buscar el mejor y más económico funcionamiento del área que le fue asignada.

A partir de Barquin (1992, 393,412), Giménez (1992, 713-714) y Temes $(1994,52-53)$, se agrupan las divisiones de una clínica de la siguiente manera:

- Secciones principales directas o sectores finales.- Son aquellas que desarrollan una actividad que, por si misma, constituye uno de los fines previstos de la clínica; son sectores de salida en los cuales deben concentrarse los gastos. Son secciones principales directas: Hospitalización, quirófano, cuidados intensivos, emergencia, sala de partos, retén, sala de quemados.

Secciones principales complementanias, paramédicas o sectores intermedios.- Son las que brindan apoyo a las tareas desarrolladas en las secciones principales directas; su ritmo y volumen de actividad están condicionados por la demanda de servicios que hacen tales secciones. Las secciones principales complementarias serian: Banco de sangre, rayos $x$, laboratorio, anatomía patológica, farmacia, fisioterapia, ultrasonido, terapia respiratoria, sala de yeso, cocina, lavandería y lencería.

Secciones auxiliares o sectores primarios.- Son aquellos que brindan apoyo de tipo general a las secciones principales, tanto directas como complementarias, para que éstas puedan desarrollar eficientemente su actividad. En otras palabras, sus prestaciones van dirigidas a otras secciones de la clínica y, por lo tanto, los desembolsos de las secciones auxiliares deben ser objeto de traspaso a las primeras. Como secciones auxiliares tenemos: Dirección, mantenimiento, facturación, cobranzas, admisión, contabilidad, informática, saneamiento, nómina, suministros.

Una vez discriminadas las divisiones o secciones de un hospital o clínica, se procedió a analizar los diversos costos causados por las mencionadas secciones.

Se observa que algunos costos se pueden asociar o identificar con el área de servicios que los origino; estos pueden y deben ser cargados directamente a esa área de servicios. Son desembolsos propios que no deben ser repartidos entre las otras áreas y son denominados costos directos. Según Horngren et al (1996, 27) están relacionados con el objeto de costos (área de servicios), y puede hacerse su seguimiento de manera económicamente factible. Ejemplo de estos costos son, sueldos y demás remuneraciones del personal asignado a la sección, depreciación de equipos y mobiliario, reparación de equipos médicos, alquiler de equipos usados, etc. Lo ideal es que el sistema contable esté diseñado para que los cargos a cada sección sean automáticos desde el sistema que controle el origen de dichos costos; por ejemplo, costos del personal desde la nómina, medicinas desde la farmacia, materiales desde el control de inventarios, depreciaciones desde el control de activos fijos, etc.

Otros costos, en cambio, deben prorratearse entre vanias secciones, de acuerdo al grado de afectación que se determine; la razón es, bien porque no se 
pueda determinar con exactitud el consumo que cada sección realiza (teléfono, agua, energía eléctrica, depreciación planta física, etc.), o bien porque son costos que la gerencia autoriza para la institución en conjunto (primas de seguros, impuestos, etc.); debe tratarse de que esta distribución se haga usando criterios de asignación razonables (representativos del costo a asignar), de fácil ejecución y de bajo costo. (Temes, 1994, 54). Estos se denominan costos indirectos y aunque están relacionados con el objeto de costos (áreas de servicios), no puede hacerse su seguimiento en forma económicamente factible; son cargados a cada sección relacionada usando un método de adjudicación de costos. (Horngren et al, 1996, 27)

De igual manera deben establecerse bases o criterios de asignación razonables para que los costos de las áreas auxiliares sean absorbidos por las secciones principales. Para Giménez (1992, 714), debido a que las secciones auxiliares tienen relación tanto con las secciones principales complementarias como con las principales directas, la asignación de costos debe hacerse en dos etapas.

El método propuesto por Giménez et al (1992, 715-716) cuenta en total, con tres etapas:

Asignación primaria.- Tanto las secciones auxiliares, como las secciones principales complementarias y directas reciben sus costos directos, a través de las cuentas establecidas en el catálogo de cuentas. En cuanto a los costos indirectos, estos son distribuidos entre las secciones usuarias teniendo como base, los criterios de asignación determinados.
Al final de esta etapa, cada una de las secciones contendrá sus costos dírectos y costos indirectos propios asignados.

Asignación secundaria.- En esta etapa, los costos de las secciones auxiliares deben ser distribuidos a las secciones principales complementarias y directas, en función del servicio prestado a cada una de ellas. Finalizada esta etapa los costos quedarán repartidos sólo entre las secciones principales complementarias y principales directas; cada una de ellas tendrá sus costos directos, costos indirectos propios asignados y costos asignados de las secciones auxiliares.

Asignación final.- Consiste en distribuir los costos de las secciones principales intermedias a las principales directas. Finalmente, los costos quedan concentrados sólo en las secciones principales directas, asi: Costos directos, costos indirectos propios asignados, costos asignados de las secciones auxiliares y costos asignados de las secciones principales complementarias.

Analizadas las secciones y los egresos por ellas causados, se puedo proceder a diseñar el catálogo de cuentas, cuya estructura debe estar en perfecta concordancia con dichas secciones o áreas de servicios. En la Tabla № 1 se observa como están conformados los códigos equivalentes a las cuentas establecidas; comprende una estructura general para todos los egresos de las áreas prestadoras de servicios y un detalle sólo para la sección de hospitalización ${ }^{2}$. La especificación de las demás secciones se deberá realizar posteriormente, ya que no es objeto de este trabajo. 


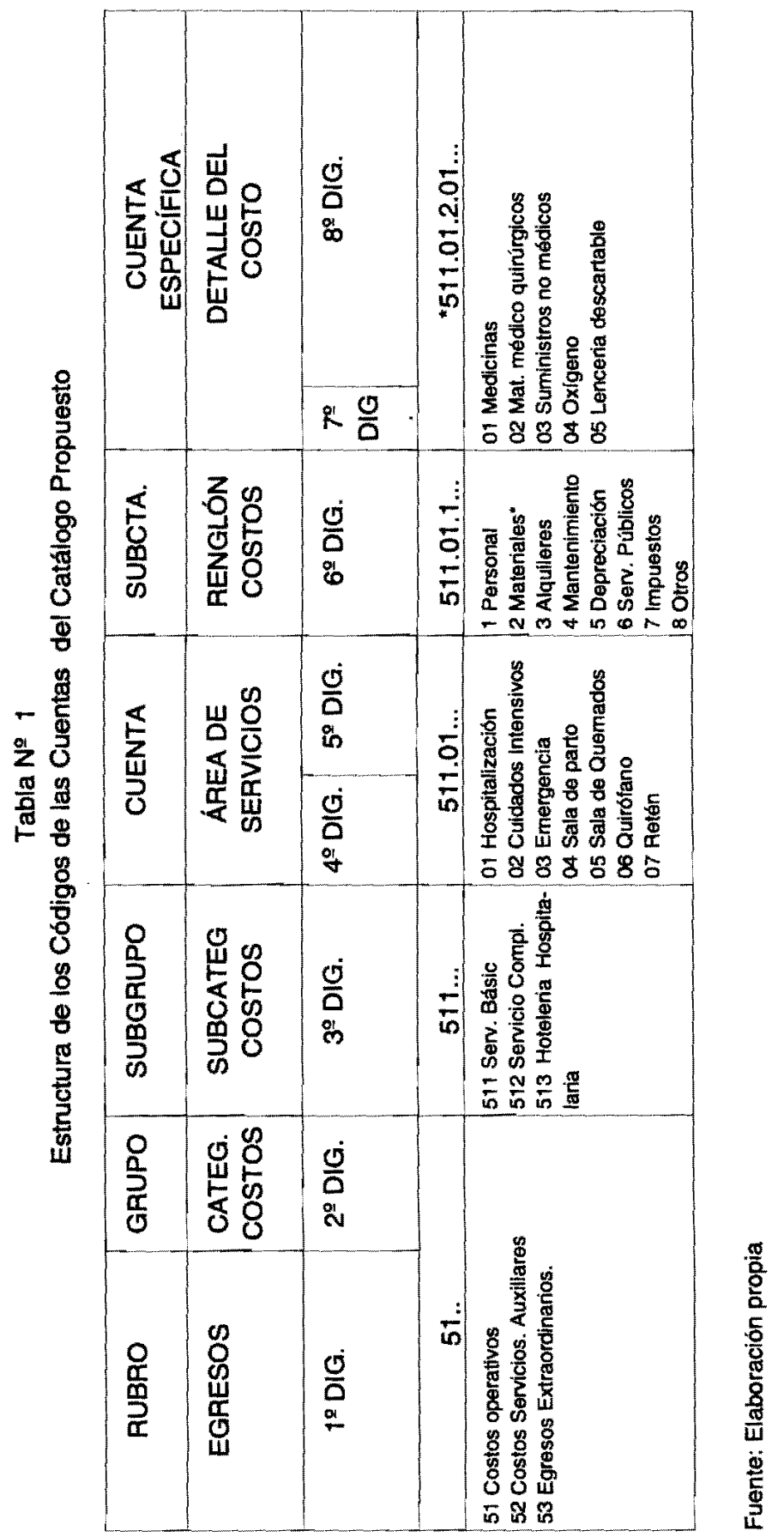




\section{Conclusiones}

Los catálogos de cuentas reflejan al menos parcialmente - el desarrollo e implantación de los sistemas contables. Su análisis, en las clínicas objeto de estudio evidencia que no son producto de un proceso consciente que implique la necesidad de conocer los costos causados por la prestación de los servicios. La importancia de los costos no ha sido apreciada en su justo valor; en consecuencia, las decisiones se toman sin contar con la adecuada información que las respalde; por lo tanto se deduce que las decisiones acertadas son más bien el resultado de la intuición de sus directivos. La contabilidad ayuda a los administradores a desarrollar sus actividades; pero los contadores deben comprender cómo aquellos utilizan la información contable, pues el éxito de la contabilidad depende de que los administradores tomen mejores decisiones gracias a la información contable recibida.

La estructura de los catálogos de cuentas no permite en ninguna de las clínicas analizadas, determinar el valor de los elementos de costos de los servicios prestados; tampoco facilita el análisis de los costos con el propósito de controlarlos y en consecuencia reducirlos; de la misma manera no sienta las bases para determinar técnicamente los precios de los servicios. En la práctica quedó demostrado que una información tan importante para una clínica como el costo cama/día de hospitalización no se puede calcular a partir de los registros contables existentes.
Sin lugar a dudas, el análisis de la utilidad potencial de los registros contables de las clínicas estudiadas, realizado a través de los grupos y subgrupos relativos a costos, evidencia una profunda contradicción entre el "deber ser" y la práctica contable, lo cual es más acentuado en el primer caso estudiado.

Tanto la diversificación de productos y servicios, como la intensidad de la competencia, requieren mejores sistemas contables. Sin embargo, esto sólo no garantiza el éxito de una empresa, el cual depende, por un lado, de que los productos y servicios satisfagan las necesidades de los clientes, y por el otro, de la razonabilidad de sus costos; en este orden de ideas, unos registros contables que permitan, entre otras cosas: controlar los costos y medir la productividad; determinar adecuadamente el costo de los servicios prestados; analizar y evaluar el desempeño de los responsables de las áreas de servicios; deben contribuir, sin duda, a que se tomen decisiones más acertadas.

A partir del análisis realizado y con el apoyo de fuentes bibliográficas especializadas, se propuso la estructura posible las cuentas de egresos, y la discriminación correspondiente al área de hospitalización, como un primer aporte para superar las deficiencias observadas.

Su factibilidad de aplicación requiere estrechar relaciones entre la Universidad y las instituciones de salud, a través del personal responsable de los sistemas contables y de los directivos, a fin de contribuir a una gestión eficiente. 


\section{Referencias Bibliográficas}

Barquin, Manuel (1992) Dirección de Hospitales. Sistemas de Atención Médica, $6^{q}$ edición, McGraw-Hill, México.

Gamboa, Teresa y Rangel, Elba (1996) Desaflos Gerenciales de los Sistemas de Salud en Colombia y Venezuela. Rev. Venez. de Gerencia, Vol. 1, No 2, Maracaibo.

Giménez, Cartos M. et al (1992), Tratado de Contabilidad de Costos. $5^{\circ}$ edición, Ediciones Macchi, Argentina.

González DE F., Alitza Y Rodríguez M., Guillermo (1996) Costos del Serviclo de Cirugía Ambulatoria basados en Actividades. Caso: Hospital Universitario de Maracaibo. La Universidad del Zulia, Facultad de Ciencias Económicas y Sociales. Trabajo de grado para optar al titulo de Magister Scientiarum en Gerencia de Empresas, mención Industrial, Maracaibo.

Horngren, Charles T., Foster, George y DATAR, Srikant M. (1996) Contabilidad de Costos. Un Enfoque Gerencial. $8^{8}$ edición, Prentice-Hall, México.

Instituto de Seguros Sociales (1980 a) Organización y Funcionamlento de la Atención de Salud. Acuerdo Ne 158 , en Atención de Salud. Ministerio de Trabajo y Seguridad Social, Repübilca de Colombia.
Irarrábal C., Anibal (1991) Principios de Contabilldad para la Gestión. 1 edición, Ediciones Universidad Católica de Chile, Santiago de Chile.

Jaramillo Pérez, Iván (1994) El Futuro de la Salud en Colombla. Política Social, Mercado y Descentralización. Coedición Fundaciones FESCOL, FES, Corona y Restrepo Barco, Bogotá.

Khadem, Riaz y Lober, Robert (1994) Administración en una Página. Cómo utilizar la información para lograr sus metas, $11^{\mathrm{D}}$ reimpresión, Editorial Norma, Bogotá, Colombia.

Ortega Pérez de LeóN, Armando (1990) Contabilidad de Costos. $4^{\text {a }}$ edición, Uteha, México.

Polimeni, Ralph S., Fabozzi, Frank J. y Adelberg, Arthur H. (1994) Contabilidad de Costos. Conceptos y Aplicaciones para la toma de Decisiones Gerencia. les. $3^{n}$ edición. McGraw-Hill, Colombia.

Ramírez Padilla, David Noel (1994) Contabilidad Administrativa, $\quad 4^{\mathbf{e}}$ edición, McGraw-Hill, México.

Shank, John K. y Govindarajan, Vijay (1995) Gerencia Estratégica de Costos. La Nueva Herramienta para Desarrollar una Ventaja Competitiva. $1^{\text {a edición. }}$ Editorial Norma, Colombia.

Temes Montes, José L., Diaz Fernández, José L. y Parra Vázquez, Blanca (1994) EI Coste por Proceso Hospitalario. 1 edición, Interamericana McGrawHill, España. 